\title{
Rotational transport via spontaneous symmetry breaking in vibrated disk packings
}

\author{
Cristian Fernando Moukarzel \\ Departamento de Física Aplicada, CINVESTAV del IPN, 97310 Mérida, Yucatán, México \\ Gonzalo Peraza-Mues \\ Universidad Politécnica de Yucatán, Carretera Mérida-Tetiz. Km 4.5, Ucú, Yucatán, México \\ Osvaldo Carvente \\ Departamento de Ingeniería Física, Universidad Autónoma de Yucatán, Mérida, Yucatán, México.
}

\begin{abstract}
It is shown that vibrated packings of frictional disks self-organize cooperatively onto a rotationaltransport state where the long-time angular velocity $\bar{\omega}_{i}$ of each disk $i$ is nonzero. Steady rotation is mediated by the spontaneous breaking of local reflection symmetry, arising when the cages in which disks are constrained by their neighbors acquire quenched disorder at large packing densities. Experiments and numerical simulation of this unexpected phenomenon show excellent agreement with each other, revealing two rotational phases as a function of excitation intensity, respectively the low-drive (LDR) and the moderate-drive (MDR) regimes. In the LDR, interdisk contacts are persistent and rotation happens due to frictional sliding. In the MDR, disks bounce against each other, still forming a solid phase. In the LDR, simple energy-dissipation arguments are provided, that support the observed dependence of the typical rotational velocity on excitation strength.
\end{abstract}

Modern civilization rests upon our ability to harness energy on large scales. The size and complexity of human societies is strongly correlated with their energy consumption rate. Population and technological complexity developed, in relative terms, only slowly, until practical thermal engines where developed [1]. Thermal engines allowed large amounts of already available fuel energy to be used to produce work. A thermal engine transforms random thermal agitation into directed motion, and is thus in essence nothing but a noise rectifier. Noise rectification is clearly a process of fundamental importance, and not only for energy generation. Much of our modern electronic technology, as well as a large number of important biological processes, depend on mechanisms that filter out noise, obtaining predictable behavior out of randomness, down to the microscopic scale. This justifies the continued interest in this field, and the search for novel noise rectification processes [2 5 ]

The second law of thermodynamics ensures that no device extracting work from random motion can operate in thermal equilibrium. Transport requires dissipation. The subtle way in which the second law constrains the behavior of even the simplest noise rectifier has been discussed in physics textbooks [6]. In addition to the breaking of time reflection-symmetry, implied by the existence of dissipation, a spatial symmetry must as well be broken for nonequilibrium transport to occur. Most nonequilibrium systems that are capable of unidirectional motion [7-9] or rotation [10 14] have, by design, a broken spatial symmetry. Such systems are said to have an explicit symmetry breaking.

Remarkably, however, some a priori spatially symmetric dissipative systems are as well able to sustain transport. Transport is made possible in these systems by the spontaneous breaking of a spatial symmetry, that is, the fact that the system becomes dynamically trapped within a non-symmetric subset of its phase-space [15].
Transport mediated by a spontaneously symmetry breaking (SSBT) is less common than that occurring on explicitly non-symmetric systems, and often requires some degree of fine tuning or cleverly targeted design [16-18]. Here, an unexpected novel example of SSBT is presented,
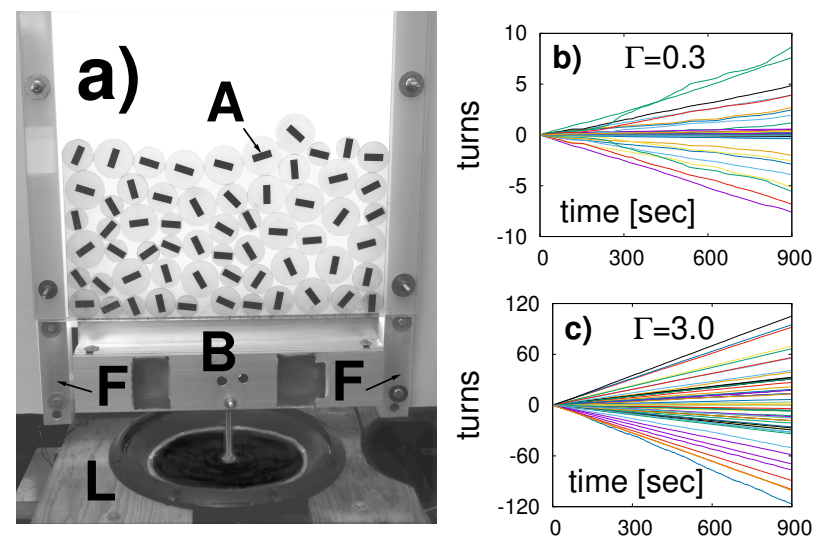

FIG. 1: a) Experimental apparatus used to study rotation in vibrated disk packings. A loudspeaker (L) rigidly connected to a movable bottom profile (B) provides the required excitation. Disks have labels (A) for motion tracking, and are confined between two glass panels rigidly fixed to the ground through metallic profiles (F). Experimental angular trajectories of all disks for a packing with (b) $\Gamma=0.3, \mathbf{c}) \Gamma=3.0$ ) show the persistence of rotation over the observation timescale of $900 \mathrm{sec}$.

that occurs in a remarkably simple system: vertically vibrated disk packings under gravity. We show, by experiment (See Video Experiment) and simulation (See Video Simulation), that at large packing densities a rotationaltransport phase appears, in which each disk rotates with nonzero long-time average angular velocity. Surprisingly, this auto-organized transport phenomenon remained unnoticed despite decades of intense work scrutinizing ex- 
ternally excited disks or spheres as models of nonequilibrium phenomena, even when rotations [19 22$]$ where specifically addressed in some cases.

Our main external control parameter is $\Gamma$, the peak bottom acceleration divided by gravity $g$. The disks, their mutual interactions, and the external driving, are reflection-symmetric. At large $\Gamma$, and therefore at low packing densities in our gravity-confined setup (in the "fluid", highly agitated phase), no rotational transport occurs, because the local environment of each disk has chiral symmetry over long times. Systematic rotation requires the dynamical breaking of this symmetry. This only happens, for the system in question, at high packing densities. In the "solid" phase that forms at high densities (small $\Gamma$ ), each disk becomes nonergodically trapped in an irregular cage formed by its neighbors 23. Individual cages are not symmetric, and this produces statistical imbalances in frictional interactions that make systematic rotation possible over long times. The fact that most earlier studies focused on the less dense fluid phase could explain why rotation had not been reported previously.

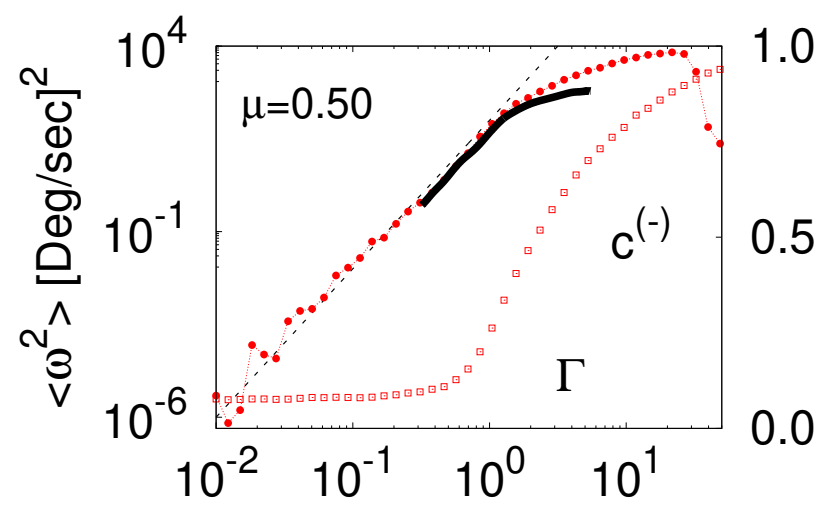

FIG. 2: (color online) The mean squared rotational velocity $\left.<\bar{\omega}^{2}\right\rangle$ (thick black line: Experiment, red full dots: Simulation) as a function of relative acceleration $\Gamma$ reveals a dynamical transition at $\Gamma \approx 1$. The nature of this transition is clarified by measuring the average contact-deficit $c^{(-)}$defined in the text (red empty squares - only in simulations). In the low-drive regime $\Gamma<1$, contacts are persistent and disks rotate via sliding. The moderate-drive regime $1<\Gamma \lesssim 30$ is a collision-dominated rotational phase. The black dashed line is $\Gamma^{4}$. At very large drives $\Gamma>30$, disk diffusion restores disk-neighborhood symmetry and $\left\langle\bar{\omega}^{2}\right\rangle \rightarrow 0$ at long times.

\section{Methods}

Experimental packings (Fig. 1a) are made of 3 sets of twenty disks each, respectively with radii 1.5, 2.0 and $2.5 \mathrm{~cm}$, machine-cut from a $3 \mathrm{~mm}$-thick acrylic sheet, with sandpaper-treated edges for increased friction, and an attached black rectangular label (A) for motion tracking. Disks are confined between two vertical glass panes $(4 \mathrm{~mm}$ separation), which are fixed to two steel frames (F) that are rigidly attached to the floor. Inserted through the 40-cm wide bottom opening, an aluminum profile (B), restricted to move vertically by a two-rail system, is rigidly connected to a loudspeaker (L) that provides the vertical vibrational excitation. The speaker is driven by a $1500 \mathrm{~W}$ amplifier, fed by a sinusoidal signal with variable intensity $S$ and fixed frequency $f_{b}=80 \mathrm{~Hz}$.

After mixing the packing, the signal intensity is set to its maximum admissible value $S=1$, and later decreases step-wise at 20-minute intervals, until $S=0.05$ is reached. A set of $M$ measurements with decreasing signal intensities $S_{m}, m=1, \ldots, M$ constitutes a run $r$ of the experiment. Within each 20-minute interval, a 5-minute relaxation period is followed by data acquisition for 15 minutes. Photos of the packing are taken at every second, and data from two adxl345 triaxial accelerometric sensors $(L, R)$, attached to opposite ends of the bottom profile, are stored at $200 \mathrm{~Hz}$. Collision noise is filtered out from sensor signals by calculating the integrals $I_{m}^{L, R}$ of their spectral densities in a $10 \mathrm{~Hz}$-wide window around $f_{b}$. Our estimators $\Gamma_{m}^{L, R}=\sqrt{2 I_{m}^{L, R}}\left(2 \pi f_{b}\right)^{2} / g$ for vertical excitation result from calculating the peak acceleration corresponding to a sinusoidal signal with spectral density integral $I_{m}^{L, R}$ and dividing it by $g$. These two estimators are then averaged to obtain $\Gamma_{m}$, verifying that their difference is never larger than a few percents. We also check that horizontal and off-plane vibrations are negligible.

Experimental data is presented from 25 runs $r=$ $1, \ldots, 25$, each taking approximately 10 hours to complete. For each run $r$ and signal strength $S_{m}$, the resulting $T=900$ images are processed [24, yielding positional and angular coordinates $\mathbf{x}_{i, t}^{r, m}, \theta_{i, t}^{r, m}$ for each disk $i$ at 1 -sec intervals $t=1, \ldots, T$.

Although the same set $\left\{S_{m}\right\}$ of amplifier signals is always used in experiments, the amplitude of motion of the bottom depends on the response of each packing. Therefore, each run $r$ results in a slightly different set of estimated vertical accelerations $\left\{\Gamma_{m}^{r}\right\}$, where $m$ indicates the excitation amplitude. To estimate the $\Gamma$ dependence of measured quantities of interest, averages are then taken over all available measurements, using a normalized Gaussian weight depending on $\left(\Gamma_{m}^{r}-\Gamma\right)$, with a narrow width $\sigma$.

In Molecular Dynamics (MD) simulation of disks, linear elasticity is assumed, and the Cundall-Strack [25] algorithm is used to calculate elastic tangential forces $\tau$. The algorithm assumes that disks have an elastic "skin" that can be tangentially stretched. Amonton's condition $|\tau| \leq \mu n$ constrains tangential forces to be smaller than the normal force $n$ times the friction coefficient $\mu$, which is appoximately 0.5 for sandpaper-treated acrylic. A skin slip occurs whenever this condition is violated, whereby some of the skin elastic energy is dissipated. A fifth-order predictor-corrector algorithm [26] integrates Newton's equations of motion in discrete time steps $\delta t=10^{-6}$ sec, which is approximately one hundredth of the smallest elastic oscillation period in the 
system. Physical parameters such as normal elastic constant, mass density, etc. where chosen to represent the acrylic-disk experimental system as closely as possible. Further details of the simulation protocol follow standard practices in MD simulations [27, 28. A total of 224 different packings were simulated with MD with physical run times of $t=5000 \mathrm{sec}$, or $5 \times 10^{9}$ integration steps, per simulation under constant conditions (the reasons for using such large simulation times are explained in Auxiliary Document 2 ). The numerical results we report here are averages over runs $r$, at constant $\Gamma$.

\section{Results}

Figures $1 \mathrm{~b}, \mathrm{c}$ show that the angular coordinate of each disk $i$ evolves almost linearly in time, with an average angular velocity given by $\bar{\omega}_{i}=\left(\theta_{i, t=T}-\theta_{i, t=0}\right) / T$. Since $\left\{\bar{\omega}_{i}\right\}$ are symmetrically distributed around zero, the typical rotational velocity at a given level of excitation $\Gamma$ can be estimated as the square root of $\left\langle\bar{\omega}^{2}\right\rangle$, where $<>$ indicates an average over all disks.

Our main results for $\left\langle\bar{\omega}^{2}\right\rangle_{(\Gamma)}$, summarized in Fig. 2 , show the existence of a dynamical transition near $\Gamma \approx 1$, with very good phenomenological agreement between experiment (thick line) and simulation (full dots). In both cases $\left\langle\bar{\omega}^{2}\right\rangle$ is found to behave as $\Gamma^{4}$ up to $\Gamma \approx 1$, which is a natural driving threshold [29] under gravity. For larger excitation intensities, $\left\langle\bar{\omega}^{2}>\right.$ grows slower with $\Gamma$. For very strong intensities $\Gamma>30$ (only in MD - See Video High Drive - this regime was not reachable in experiments), the packing undergoes vibrofluidization, disks execute unbiased rotational random walks, and $<\bar{\omega}^{2}>\rightarrow 0$ in the long run. We verified that the excitation intensity at which this happens roughly coincides with the appearance of diffusive behavior in disk centers.

Numerical simulation provides access to contact details, which allows us to get useful insight into the nature of the dynamical transition seen at $\Gamma \approx 1$. We measure the Contact Deficit, defined here as $c^{(-)}=1-N_{\text {contacts }} /\left(2 N_{\text {disks }}\right)$, where $N_{\text {contacts }}$ is the number of disk contacts (walls included) at a given instant, and $2 N_{\text {disks }}$ is the isostatic [30] number of contacts that defines a two-dimensional rigid packing in static equilibrium. The Contact Deficit $c^{-}$is zero for a static rigid packing under gravity (in the limit of rigid, undeformable disks [30]), and equals one for a diluted granular gas with no contacts. Its average value, obtained from MD simulations (Fig. 2, empty squares) remains very small in the low-intensity regime $\Gamma \lesssim 1$, growing steeply for $\Gamma \gtrsim 1$. This shows that in the lowdrive regime the maximum possible number $2 N_{\text {disks }}$ of interdisk contacts remain closed most of the time. The moderate-drive $\Gamma \gtrsim 1$ phase is, on the other hand, a "collisional" phase where contacts occurr only briefly so $N_{\text {contacts }}<<2 N_{\text {disks }}$.

Armed with this knowledge, we can further our theoretical understanding of the dynamical behavior at low excitation intensities. In the low-drive regime $\Gamma \lesssim 1$, an energy-dissipation argument can be built, that supports the $\left\langle\bar{\omega}^{2}>\propto \Gamma^{4}\right.$ relationship observed in Fig. 2 for experiments and simulation (See Auxiliary Document 1). The power dissipated by the packing depends, among other parameters, on material properties such as friction, elastic constants, and viscoelasticity, but we will only be interestd in its $\Gamma$-dependence. At constant driving frequency $f_{b}$, the bottom, moving with amplitude $A_{b}$, injects an amount of power $P_{I}=C_{v}\left(f_{b} A_{b}\right)^{2}$ into the packing, with $C_{v}$ an effective viscous coefficient depending on material properties of the packing. This power is dissipated, in general, through inelastic collisions and frictional tangential forces $\tau$ among disks. In the persistentcontact regime, however, dissipation due to inelastic collisions can be neglected. The dominant dissipation mechanism is in this case frictional, i.e. due to contact slips. The global rate of dissipation $P_{d}$ is thus roughly proportional to the average rate of tangential slip times the average interdisk tangential force. The average tangential force equals $\mu$, the frictional coefficient, times the average normal force. The globally averaged tangential slip per unit time can be assumed to be proportional to the typical rotational velocity, which is given by $\sqrt{\left\langle\bar{\omega}^{2}\right\rangle}$. The dissipated power $\sim A_{b}^{2}$ is thus roughly proportional to $\tau_{\text {avg }} \sqrt{\left\langle\bar{\omega}^{2}\right\rangle}=\mu n_{\text {avg }} \sqrt{\left\langle\bar{\omega}^{2}\right\rangle}$, where $n_{\text {avg }}$ is the timeaveraged normal force between two disks, and averaged over pairs of disks in contact. Now, it is known that the hydrostatic hypothesis is valid for excited granular systems 31] such as ours. Therefore, interdisk normal forces are dictated by the weight of the layers above a given pair, and thus do not vary with $\Gamma$. Equating dissipated with injected power, using the fact that $\Gamma \propto A_{b}$ and focusing on $\Gamma$-dependences only, one obtains $\left\langle\bar{\omega}^{2}>\propto A_{b}^{4} \propto \Gamma^{4}\right.$, as observed in the low-drive regime for both experiments and simulation (See Fig. 2). This approach ceases to be useful in the bouncing regime $\Gamma \gtrsim 1$, where free-flight rotation can no longer be linked to dissipation.

By measuring the distance dependence of the two-disk angular velocity correlation functions, we find that neighboring disks have a statistical tendency to rotate in opposite directions, as favored by frictional constraints. This of course does not imply perfect slip-less rotation, which is only possible on arrays specifically designed [32] for that purpose. As a further consistency test for numerical results, Event-driven (ED) simulations [28] where also conducted for comparison with MD. ED simulations only make sense at not too small driving intensities $(\Gamma>2$ in this case), so that persistent contacts do not exist. We found a very good coincidence of results between these two simulational methods, in the driving regime where both MD and ED can be applied.

Given that our MD implementation is well validated by our own experimental and ED simulation studies, we can use it as an exploratory tool for more general setups that are not easily accesible to experimentation. We did, for example, MD simulations of vibrated elastic frictional packings with periodic boundary conditions across 
the horizontal direction. The rotational properties discussed here remain basically unchanged. We then conclude that rotation is not wall-induced. Other kinds of bottom excitation mechanisms were also explored in simulations, as for example applying random uncorrelated bumps on each disk of the lowest layer. These showed that rotational organization does not depend on the periodic coordinated motion of the bottom. The rotational phenomenon reported here was also found numerically in closely confined packings without gravity, when these are excited either by vibrating all four boundary walls or via Langevin noise (random point forces - no torques) acting on disk centers. This demonstrates that the rotational organization phenomenon is cooperatively induced by frictional interactions among disks and does not depend on the existence of a preferred gravity direction. We plan to study horizontally confined 2D packings experimentally in the near future. More details on this rotational phenomenon will be provided somewhere else for reasons of space.

We have made some analytical progress as well, for highly simplified situations. We studied [33, 34] threedisk toy models [35] or billiards, consisting of one freely moving disk supported against gravity by two vibrating ones. For these, rotation invariably appears whenever the system is tilted with respect to gravity, thus explicitely breaking reflection symmetry. Rotation is seen in both the persistent-contact 34. (low drive) and bouncing 33. (moderate drive) regimes, which are also observed for this simplified toy model.

In summary, we report a spontaneous-rotation phenomenon in vibrated disk-packings under gravity. Disorder in disk neighborhoods, in the solid phase, locally breaks reflection symmetry, allowing for persistent unidirectional rotation of disks. A similar rotational transport phenomenon is observed in confined two-dimensional packings without gravity. Based on our present understanding of this auto-organized rotational state, we believe that a similar phenomenon should be observed in sphere packings as well. This spontaneous rotation phenomenon is potentially relevant for colloids, cellular systems, and other close-packed systems of approximately circular or spherical shape.

GPM was supported by a $\mathrm{PhD}$ fellowship from CONACYT México. We are grateful for the continued use of extensive computational resources on clusters "Xiuhcoatl" and "Kukulcán" of CINVESTAV. Ana María Vidales and Aldo H. Romero-Castro helped in the early numerical stages of this work. Their contributions are gratefully acknowledged.
[1] Temis G. Taylor and Joseph A. Tainter. The nexus of population, energy, innovation, and complexity. American Journal of Economics and Sociology, 75(4):10051043, 2016.

[2] M. C. Cross and P. C. Hohenberg. Pattern formation outside of equilibrium. Rev. Mod. Phys., 65:851-1112, Jul 1993.

[3] T. Ross Kelly, Harshani De Silva, and Richard A. Silva. Unidirectional rotary motion in a molecular system. $\mathrm{Na}$ ture, 401:150-152, September 1999.

[4] Peter Reimann. Brownian motors: noisy transport far from equilibrium. Physics Reports, 361(2-4):57-265, April 2002.

[5] P. Reimann and P. Hänggi. Introduction to the physics of Brownian motors. Applied Physics A, 75(2):169-178, January 2014.

[6] Richard P. Feynman, Robert B. Leighton, and Matthew Sands. The Feynman Lectures on Physics, volume 1. Basic books, 2011.

[7] Marcelo O. Magnasco. Forced thermal ratchets. Physical Review Letters, 71(10):1477-1481, September 1993.

[8] A. Baule and P. Sollich. Singular features in noiseinduced transport with dry friction. EPL (Europhysics Letters), 97(2):20001, 2012.

[9] S M Simon, C S Peskin, and G F Oster. What drives the translocation of proteins? Proceedings of the National Academy of Sciences, 89(9):3770-3774, 1992.

[10] J.-C. Tsai, Fangfu Ye, Juan Rodriguez, J. P. Gollub, and T. C. Lubensky. A chiral granular gas. Phys. Rev. Lett., 94:214301, May 2005.

[11] Peter Eshuis, Ko van der Weele, Detlef Lohse, and Devaraj van der Meer. Experimental Realization of a Rota- tional Ratchet in a Granular Gas. Physical Review Letters, 104(24):248001, June 2010.

[12] Ernesto Altshuler, Jose Martin Pastor, Angel Garcimartín, Iker Zuriguel, and Diego Maza. Vibrot, a Simple Device for the Conversion of Vibration into Rotation Mediated by Friction: Preliminary Evaluation. PLOS ONE, 8(8):e67838, August 2013.

[13] Benjamin C. van Zuiden, Jayson Paulose, William T. M. Irvine, Denis Bartolo, and Vincenzo Vitelli. Spatiotemporal order and emergent edge currents in active spinner materials. Proceedings of the National Academy of Sciences, 113(46):12919-12924, November 2016.

[14] Christian Scholz, Michael Engel, and Thorsten Pöschel. Rotating robots move collectively and self-organize. $\mathrm{Na}$ ture Communications, 9(1):931, 2018.

[15] É. Roldán, I. A. Martínez, J. M. R. Parrondo, and D. Petrov. Universal features in the energetics of symmetry breaking. Nature Physics, 10(6):457-461, 2014.

[16] Frank Jülicher and Jacques Prost. Cooperative molecular motors. Phys. Rev. Lett., 75:2618-2621, Sep 1995.

[17] P Reimann, R Kawai, C. Van den Broeck, and P Hänggi. Coupled brownian motors: Anomalous hysteresis and zero-bias negative conductance. Europhysics Letters (EPL), 45(5):545-551, mar 1999.

[18] Devaraj van der Meer, Peter Reimann, Ko van der Weele, and Detlef Lohse. Spontaneous ratchet effect in a granular gas. Phys. Rev. Lett., 92:184301, May 2004.

[19] Sean McNamara and Stefan Luding. Energy nonequipartition in systems of inelastic, rough spheres. Phys. Rev. E, 58:2247-2250, Aug 1998.

[20] Olaf Herbst, Raffaele Cafiero, Annette Zippelius, Hans Jürgen Herrmann, and Stefan Luding. A driven 
two-dimensional granular gas with Coulomb friction. Physics of Fluids, 17(10):107102, October 2005.

[21] N. V. Brilliantov, T. Pöschel, W. T. Kranz, and A. Zippelius. Translations and rotations are correlated in granular gases. Phys. Rev. Lett., 98:128001, Mar 2007.

[22] Kiri Nichol and Karen E. Daniels. Equipartition of Rotational and Translational Energy in a Dense Granular Gas. Physical Review Letters, 108(1):018001, January 2012.

[23] P. M. Reis, R. A. Ingale, and M. D. Shattuck. Caging dynamics in a granular fluid. Phys. Rev. Lett., 98:188301, Apr 2007.

[24] Johannes Schindelin, Ignacio Arganda-Carreras, Erwin Frise, Verena Kaynig, Mark Longair, Tobias Pietzsch, Stephan Preibisch, Curtis Rueden, Stephan Saalfeld, Benjamin Schmid, Jean-Yves Tinevez, Daniel James White, Volker Hartenstein, Kevin Eliceiri, Pavel Tomancak, and Albert Cardona. Fiji: an open-source platform for biological-image analysis. Nature Methods, 9(7):676682, July 2012.

[25] P. A. Cundall and O. D. L. Strack. A discrete numerical model for granular assemblies. Géotechnique, 29(1):4765, March 1979.

[26] C. William Gear. The automatic integration of ordinary differential equations. Communications of the ACM, 14(3):176-179, 1971.

[27] Daan Frenkel and Berend Smit. Understanding Molecular Simulation: From Algorithms to Applications. Academic Press, edición: 2 edition, October 2001.

[28] Thorsten Pöschel and Thomas Schwager. Computational
Granular Dynamics. Springer-Verlag, Berlin/Heidelberg, 2005.

[29] Peter Eshuis, Ko van der Weele, Devaraj van der Meer, Robert Bos, and Detlef Lohse. Phase diagram of vertically shaken granular matter. Physics of Fluids, 19(12):123301, December 2007.

[30] C. F. Moukarzel. Isostatic phase transition and instability in stiff granular materials. Phys. Rev. Lett., 81:16341637, 1998.

[31] M. Yasinul Karim and Eric I. Corwin. Eliminating friction with friction: $2 \mathrm{~d}$ janssen effect in a friction-driven system. Phys. Rev. Lett., 112:188001, May 2014.

[32] D. V. Stäger, N. A. M. Araújo, and H. J. Herrmann. Prediction and Control of Slip-Free Rotation States in Sphere Assemblies. Physical Review Letters, 116(25):254301, June 2016.

[33] G. G. Peraza-Mues, Osvaldo Carvente, and Cristian F. Moukarzel. Rotation in a gravitational billiard. International Journal of Modern Physics C, page 1750021, September 2016.

[34] Gonzalo G Peraza-Mues and Cristian F Moukarzel. Sustained rotation in a vibrated disk with asymmetric supports. Journal of Statistical Mechanics: Theory and Experiment, 2019(10):103201, oct 2019.

[35] Sean McNamara, Ramón García-Rojo, and Hans Herrmann. Indeterminacy and the onset of motion in a simple granular packing. Physical Review E, 72(2):021304, August 2005. 